\title{
Extratos metanólico e acetato de etila de Solanum megalonyx Sendtn. (Solanaceae) apresentam atividade espasmolítica em íleo isolado de cobaia: um estudo comparativo
}

\author{
Rita de Cássia M. Oliveira ${ }^{1,2}$, Fabio de S. Monteiro ${ }^{1}$, Joelmir L.V. Silva ${ }^{1}$, Luciano A.A. \\ Ribeiro ${ }^{1}$, Rosemeire F. Santos ${ }^{1}$, Roberto J.B. Nascimento ${ }^{1}$, José C. Duarte ${ }^{1}$, Maria de F. Agra', \\ Tania M.S. Silva ${ }^{1}$, Fernanda R.C. Almeida ${ }^{2}$ Bagnolia A. Silva ${ }^{1}$ \\ ${ }^{1}$ Laboratório de Tecnologia Farmacêutica "Prof. Delby Fernandes de Medeiros", Universidade Federal da \\ Paraíba; Caixa Postal 5009, 58051-970, João Pessoa, PB, Brasil, \\ ${ }^{2}$ Núcleo de Pesquisas em Plantas Medicinais, Centro de Ciências da Saúde, Universidade Federal do Piauí, \\ 64049-550, Teresina, PI, Brasil
}

\begin{abstract}
RESUMO: Solanum megalonyx Sendtn. (Solanaceae) é conhecida popularmente por "jurubeba" no Nordeste do Brasil e se apresenta na forma de arbusto. Várias espécies de Solanum apresentam efeito espasmolítico em órgãos isolados. Assim, objetivou-se investigar e comparar o efeito dos extratos metanólico (SM-MeOH) e acetato de etila (SM-AcOEt), obtidos das partes aéreas de $S$. megalonyx, em íleo isolado de cobaia. SM-MeOH e SM-AcOEt antagonizaram $(\mathrm{n}=5)$ as contrações fásicas induzidas por $1 \mu \mathrm{M}$ de acetilcolina $\left(\log \mathrm{CI}_{50}=3,2 \pm 0,1\right.$ e $1,8 \pm 0,6 \mu \mathrm{g} / \mathrm{mL}$, respectivamente) ou de histamina $\left(\log \mathrm{CI}_{50}=2,8 \pm 0,5\right.$ e $1,7 \pm 0,3 \mu \mathrm{g} / \mathrm{mL}$, respectivamente). SM-MeOH e SM-AcOEt também relaxaram $(\mathrm{n}=5)$ o íleo pré-contraído por $40 \mathrm{mM}$ de $\mathrm{KCl}\left(\log \mathrm{E}_{50}=1,9 \pm 0,09\right.$ e $1,9 \pm$ $0,1 \mu \mathrm{g} / \mathrm{mL}$, respectivamente), por $1 \mu \mathrm{M}$ de histamina $\left(\log \mathrm{CE}_{50}=1,9 \pm 0,07\right.$ e $1,7 \pm 0,08 \mu \mathrm{g} / \mathrm{mL}$, respectivamente) ou de acetilcolina ( $\log \mathrm{CE}_{50}=1,9 \pm 0,02$ e $1,7 \pm 0,09 \mu \mathrm{g} / \mathrm{mL}$, respectivamente) de maneira dependente de concentração e equipotente. Demonstra-se pela primeira vez que $S$. megalonyx apresenta efeito espasmolítico não seletivo em íleo isolado de cobaia, sugerindo que os extratos podem estar agindo em um passo comum da via de sinalização dos agentes contráteis testados.
\end{abstract}

Unitermos: Solanum megalonyx, Solanaceae, íleo de cobaia, atividade espasmolítica.

\begin{abstract}
Methanol and ethyl acetate extracts from Solanum megalonyx Sendtn. (Solanaceae) present spasmolytic activity in guinea-pig ileum: a comparative study". Solanum megalonyx Sendtn. (Solanaceae) is known popularly as "jurubeba" in Northeastern Brazil where it can be found as a shrub. Several species of Solanum present spasmolytic effect in several tissues, thus this study was aimed to investigate and compare the effect of the methanol extract (SM$\mathrm{MeOH}$ ) and ethyl acetate extract (SM-AcOEt), obtained from aerial parts of Solanum megalonyx Sendtn., in guinea-pig ileum. In this work, both SM-MeOH and SM-AcOEt antagonized the phasic contraction induced by acetylcholine $1 \mu \mathrm{M}\left(\operatorname{logIC} \mathrm{I}_{50}=3.2 \pm 0.1\right.$ and $\left.1.8 \pm 0.6 \mu \mathrm{g} / \mathrm{mL}\right)$ and histamine $1 \mu \mathrm{M}\left(\operatorname{logIC} C_{50}=2.8 \pm 0.5\right.$ and $1.7 \pm 0.3 \mu \mathrm{g} / \mathrm{mL}$, respectively $)(\mathrm{n}=5)$, without statistical differences between these values. In another set of experiments, SM-MeOH and SM-AcOEt also relaxed the isolated guinea-pig ileum pre-contracted by $\mathrm{KCl} 40 \mathrm{mM}\left(\operatorname{logEC} \mathrm{C}_{50}=1.9 \pm 0.09\right.$ and 1.9 $\pm 0.1 \mu \mathrm{g} / \mathrm{mL}$, respectively), histamine $1 \mu \mathrm{M}\left(\operatorname{logEC} \mathrm{C}_{50}=1.9 \pm 0.07 \mu \mathrm{g} / \mathrm{mL}\right.$ and $1.7 \pm 0.08 \mu \mathrm{g} / \mathrm{mL}$, respectively) or acetylcholine ( $\log \mathrm{EC}_{50}=1.9 \pm 0.02 \mu \mathrm{g} / \mathrm{mL}$ and $1.7 \pm 0.09 \mu \mathrm{g} / \mathrm{mL}$, respectively) ( $\mathrm{n}$ $=5$ ) in a concentration-dependent and equipotent manner. This study demonstrates for the first time that aerial parts of $S$. megalonyx present a non-selective spasmolytic effect in guinea-pig ileum, suggesting that the extracts could be acting in a common step of the pathway signaling that leads to contraction induced by the contractile agents tested.
\end{abstract}

Keywords: Solanum megalonyx, Solanaceae, guinea-pig ileum, spasmolytic activity.

\section{INTRODUÇÃO}

$\mathrm{Na}$ vegetação do Nordeste Brasileiro estão presentes várias famílias, entre elas a Solanaceae que é rica em metabólitos secundários ativos (Barbosa-Filho et al., 1991; Silva et al., 2002; Braga et al., 2003; Silva et al., 2005). O gênero Solanum produz uma grande variedade de saponinas esteroidais e glicoalcalóides de importância na resistência natural destas plantas contra muitas pestes (Friedman et al., 1991 ). Este gênero é um dos mais amplos do reino vegetal com cerca de 1250 espécies (Nee, 2001) e tem sido extensivamente estudado, devido principalmente as atividades biológicas apresentadas por muitas de suas espécies (Ribeiro et al., 2002; Barbosa- 
Filho et al., 2005; Barbosa-Filho et al., 2006).

No Brasil algumas espécies do gênero Solanum (S. paniculatum L., S. melongena L. e S. stipulaceum Roem \& Schult.) foram relatadas por induzirem hipotensão em ratos (Almeida et al., 1984; Ribeiro et al., 1986; Shum; Chiu, 1991; Ribeiro, 2001). Várias outras espécies também apresentaram efeito espasmolítico significante como exemplo: $S$. indicum L., S. torvum Sw., S. melongena L. e $S$. dulcamara L. (Boyd, 1928; Bhakuni et al., 1969; Abraham et al., 1986; Shum; Chiu, 1991).

Desde 1999 vem sendo desenvolvido, pelo nosso grupo de pesquisa, estudos de monitoração da atividade espasmolítica de extratos e partições (fases) obtidos de algumas espécies de Solanum do Nordeste Brasileiro à saber: $S$. paludosum Moric, $S$. jabrense Agra $\&$ Nee, S. agrarium Sendtn. e S. stipulaceum Roem \& Schult (Silva et al., 2000; Claudino et al., 2001, Claudino, 2003; Cavalcante, 2001; Santos et al., 2003). Sabendose que outras espécies do gênero Solanum apresentam grande potencial farmacológico e terapêutico, o objetivo desse trabalho foi investigar a atividade espasmolítica de Solanum megalonyx Sendtn., uma espécie típica do Nordeste Brasileiro, em íleo isolado de cobaia.

\section{MATERIAL E MÉTODOS}

\section{Material botânico}

S. megalonyx Sendtn. foi coletada em abril de 2002 na Chapada Diamantina no estado da Bahia e determinada pela Dra. Maria de Fátima Agra. Uma exsicata está depositada no Herbário Prof. Lauro Pires Xavier (JPB/UFPB) e duplicatas estão depositadas na coleção de referência do Laboratório de Tecnologia Farmacêutica Prof. Delby Fernandes de Medeiros (LTF)/ UFPB, com Voucher Agra $n^{\circ} 5177$. Após coleta, folhas e partes aéreas foram secas em estufa com circulação de ar, à temperatura de $40{ }^{\circ} \mathrm{C}$, e trituradas em moinho de facas. Posteriormente, o material triturado foi extraído com metanol (PA) e acetato de etila (PA), em aparelho de Soxhlet, até o esgotamento, e seco em evaporador rotatório.

\section{Animais}

Foram utilizadas cobaias (Cavia porcellus) de ambos os sexos pesando entre $350-500 \mathrm{~g}$, provenientes do Biotério Prof. Thomas George (LTF/UFPB). Antes dos experimentos os animais eram mantidos sob rigoroso controle alimentar, com uma dieta balanceada a base de ração tipo "pellets" (Purina) com livre acesso à água, com ventilação e temperatura $\left(22 \pm 1^{\circ} \mathrm{C}\right)$ controladas e constantes. Todos os procedimentos experimentais foram aprovados pelo Comitê de Experimentação Animal da UFPB (Parecer 0512/2005).

\section{Substâncias e sais}

A acetilcolina, histamina, cloreto de cálcio dihidratado $\left(\mathrm{CaCl}_{2} \cdot 2 \mathrm{H}_{2} \mathrm{O}\right)$, cloreto de sódio $(\mathrm{NaCl})$, glicose $\left(\mathrm{C}_{6} \mathrm{H}_{12} \mathrm{O}_{6}\right)$ e fosfato monossódico 1-hidratado $\left(\mathrm{NaH}_{2} \mathrm{PO}_{4}\right.$. $\mathrm{H}_{2} \mathrm{O}$ ) foram obtidos da Merck (Brasil). O cremofor foi obtido da Sigma-Aldrich (EUA); cloreto de potássio $(\mathrm{KCl})$, bicarbonato de sódio $\left(\mathrm{NaHCO}_{3}\right)$ e sulfato de magnésio hepta-hidratado $\left(\mathrm{MgSO}_{4} \cdot 7 \mathrm{H}_{2} \mathrm{O}\right)$ foram obtidos do REAGEN (Brasil).

\section{Solução fisiológica}

Foi utilizada a solução fisiológica de Krebs modificado (Sun; Benishin, 1994) com a seguinte composição em mM: $\mathrm{NaCl}(117,0) ; \mathrm{NaHCO}_{3}(25,0)$; $\mathrm{NaH}_{2} \mathrm{PO}_{4} \cdot \mathrm{H}_{2} \mathrm{O}(1,2) ; \mathrm{CaCl}_{2} \cdot 2 \mathrm{H}_{2} \mathrm{O}(2,5) ; \mathrm{KCl} \quad(4,7)$; $\mathrm{MgSO}_{4} \cdot 7 \mathrm{H}_{2} \mathrm{O}(1,3)$ e glicose $(11,0)$ aerada com mistura carbogênica ( $95 \%$ de $\mathrm{O}_{2}$ e $5 \%$ de $\mathrm{CO}_{2}$ ) e pH ajustado para 7,4 com $\mathrm{HCl} 1 \mathrm{~N}$.

\section{Aparelhos}

Para registro das contrações isométricas, utilizouse transdutores de força isométricos 7003 acoplados a um polígrafo modelo GEMINI 7070 (UGO BASILE, Itália) e transdutores de força isométricos FORT-10 conectados a um amplificador modelo TMB4M (World Precision Instruments, EUA), ligados a um computador utilizando o programa BioMed (BioData, Brasil). As contrações isotônicas foram registradas por uma alavanca isotônica de inscrição frontal em cilindros esfumaçados de quimógrafos (DTF, Brasil). Os valores de $\mathrm{pH}$ eram verificados através de um pHmetro digital PG 2000 (GEHAKA, Brasil).

\section{Preparação dos extratos para os ensaios farmacológicos}

Os extratos foram solubilizados em cremofor (3 $\%, \mathrm{v} / \mathrm{v})$ e diluídos em água destilada $(\mathrm{pH} 7,0)$ para obtenção da solução estoque $(10 \mathrm{mg} / \mathrm{mL})$ que era estocada a $0{ }^{\circ} \mathrm{C}$. No momento da realização dos experimentos diluía-se esta solução de acordo com a exigência do protocolo. A concentração final de cremofor na cuba nunca excedeu $0,01 \%$. Nesta concentração, o cremofor foi desprovido de efeito próprio.

Efeito dos extratos SM-MeOH e SM-AcOEt frente às contrações fásicas induzidas por $1 \mu \mathrm{M}$ de acetilcolina e de histamina em íleo isolado de cobaia

Os animais eram mantidos em jejum por 18 horas (dando-lhe somente água nesse período) e sacrificados por deslocamento cervical. O abdômen era aberto e um segmento do íleo de aproximadamente $15 \mathrm{~cm}$ de comprimento, era retirado e colocado em uma placa de Petri contendo solução fisiológica de Krebs modificado a 
A

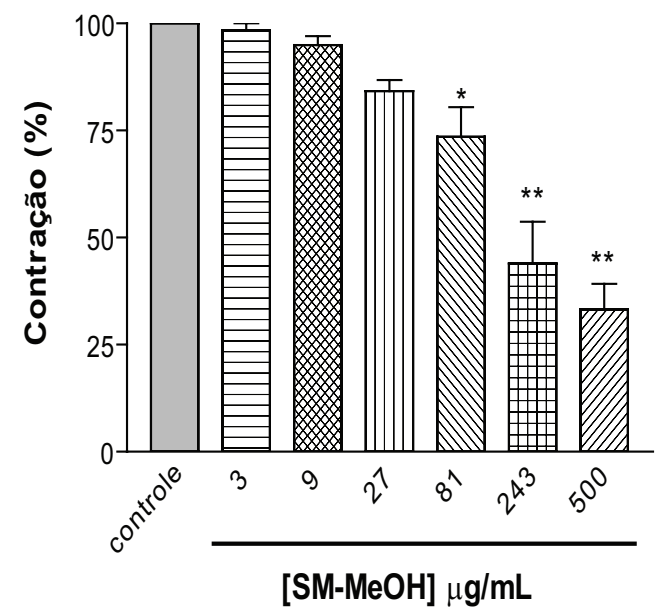

C

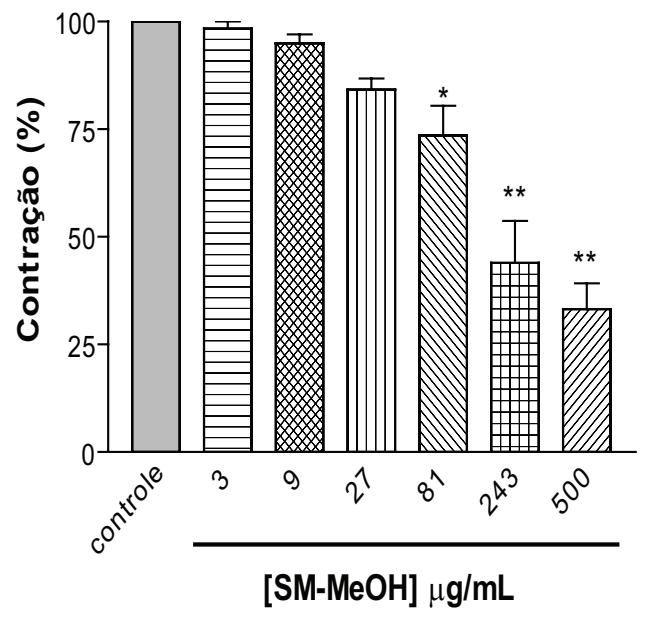

B

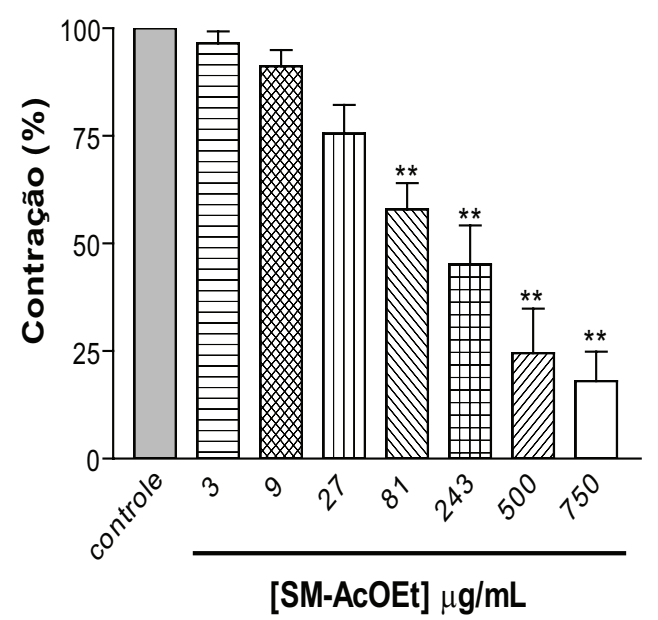

D

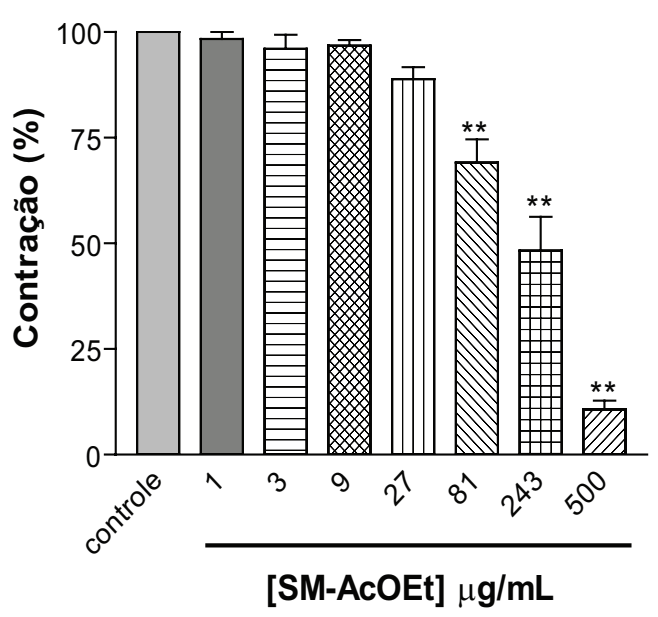

Figura 1. Efeito dos extratos SM-MeOH $(\mathbf{A}$ e $\mathbf{C})$ e SM-AcOEt $(\mathbf{B}$ e $\mathbf{D})$ frente às contrações fásicas $(\mathrm{n}=5)$ induzidas por $1 \mu \mathrm{M}$ de acetilcolina (A e B) e de histamina $(\mathbf{C}$ e D) em íleo isolado de cobaia. Colunas e barras verticais representam a média \pm e.p.m. ANOVA "one-way" seguido do teste de Bonferroni, *p $<0,05$ e **p $<0,001$ (controle $\mathrm{x}$ extrato).

$37^{\circ} \mathrm{C}$. Após cuidadosa dissecação, o segmento do íleo era seccionado em fragmentos de 2 a $3 \mathrm{~cm}$ de comprimento, suspensos individualmente em cubas de vidro $(6 \mathrm{~mL}) \mathrm{e}$ deixados em repouso por $30 \mathrm{~min}$. Após esse período de estabilização eram obtidas pelo menos duas contrações isotônicas de amplitude similares induzidas por acetilcolina ou histamina e consideradas como controle $(100 \%)$. Os extratos eram incubados em concentrações diferentes $(1,3,9,27,81,243,500$ e $750 \mu \mathrm{g} / \mathrm{mL})$ em preparações diferentes, e após 15 min., na presença dos extratos, era induzida uma nova contração pelo agonista. Os efeitos inibitórios exercidos pelos extratos foram comparados com as respostas obtidas antes de sua adição à cuba (controle).

Efeito dos extratos SM-MeOH e SM-AcOEt sobre as contrações tônicas induzidas por $\mathrm{KCl}$, acetilcolina ou histamina em íleo isolado de cobaia

Após o período de estabilização sob as condições anteriormente descritas, duas contrações isométricas sub-máximas similares para $40 \mathrm{mM}$ de $\mathrm{KCl}, 1 \mu \mathrm{M}$ de acetilcolina e de histamina eram obtidas. Durante a fase tônica sustentada da segunda resposta, o extrato era 

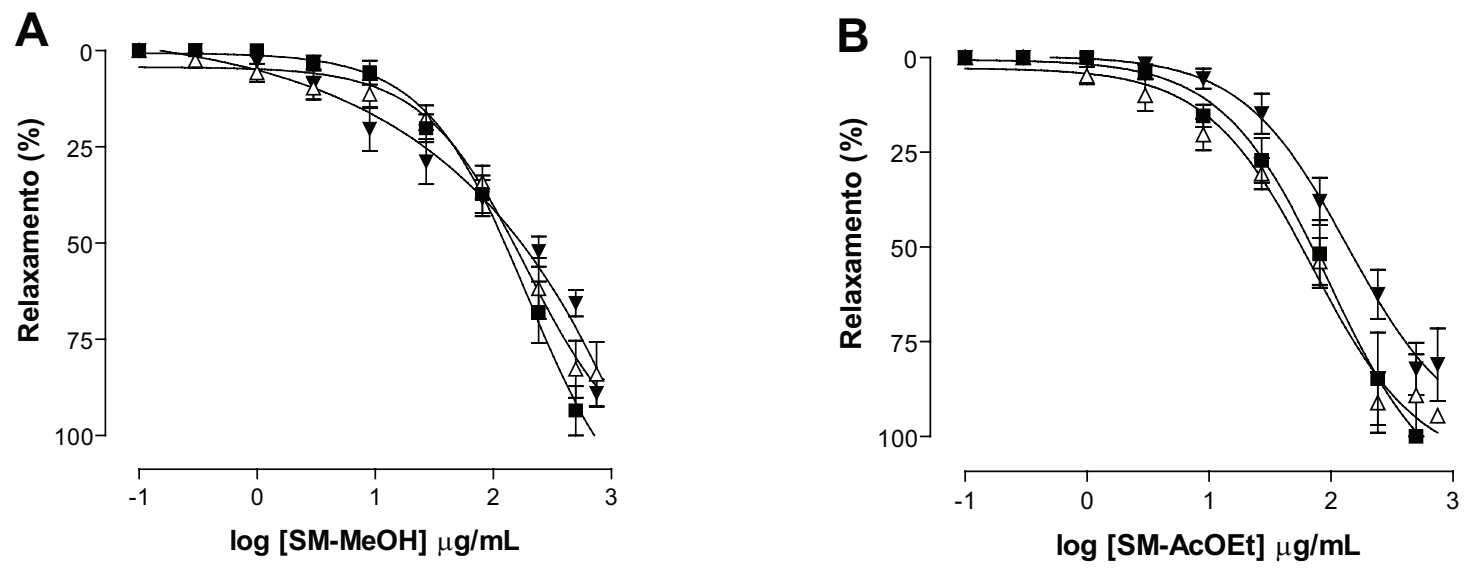

Figura 2. Efeito dos extratos $\mathrm{SM}-\mathrm{MeOH}(\mathbf{A})$ e SM-AcOEt (B) sobre as contrações tônicas induzidas por $40 \mathrm{mM}$ de $\mathrm{KCl}(\boldsymbol{\nabla}), 1$ $\mu \mathrm{M}$ de histamina $(\triangle)$ ou de acetilcolina $(\boldsymbol{\square})$, em íleo isolado de cobaia $(\mathrm{n}=5)$. Os símbolos e barras verticais representam a média \pm e.p.m., respectivamente.

adicionado de maneira cumulativa $(0,1-750 \mu \mathrm{g} / \mathrm{mL})$. O relaxamento foi expresso como a percentagem reversa da amplitude da contração inicial produzida pelos agentes contráteis.

\section{Análise estatística}

Os resultados obtidos foram expressos como média \pm erro padrão da média $(\mathrm{X} \pm$ e.p.m.) e a diferença entre as médias foram analisados estatisticamente empregando-se o teste " $\mathrm{t}$ " de Student (não-pareado) ou ANOVA "one-way" com o pós-teste de Bonferroni, onde os valores de $p<0,05$ foram considerados significantes. Os valores de $\log \mathrm{CE}_{50}$ (logaritmo na base 10 da concentração do extrato que produz uma resposta de $50 \%$ de seu efeito máximo) e $\log \mathrm{CI}_{50}$ (logaritmo na base 10 da concentração do extrato que inibe $50 \%$ da resposta máxima de um agonista) (Jenkinson et al., 1995) foram calculados por regressão não-linear. Todos os dados foram analisados usando o programa GraphPad Prism $^{\odot}$ versão 3.03 .

\section{RESULTADOS}

Efeito dos extratos SM-MeOH e SM-AcOEt frente às contrações fásicas induzidas por $1 \mu \mathrm{M}$ de acetilcolina ou de histamina em íleo isolado de cobaia

Tanto o extrato SM-MeOH $(3,0-500 \mu \mathrm{g} / \mathrm{mL})$ como o SM-AcOEt $(1,0-750 \mu \mathrm{g} / \mathrm{mL})$ antagonizaram $(\mathrm{n}=5)$ as contrações fásicas induzidas por $1 \mu \mathrm{M}$ de acetilcolina $\left(\log \mathrm{CI}_{50}=3,2 \pm 0,1\right.$ e $1,8 \pm 0,6 \mu \mathrm{g} / \mathrm{mL}$, respectivamente) (Figura $1 \mathrm{~A}$ e $\mathrm{B}$ ), ou de histamina $\left(\log \mathrm{CI}_{50}=2,8 \pm 0,5\right.$ e $1,7 \pm 0,3 \mu \mathrm{g} / \mathrm{mL}$, respectivamente) (Figura $1 \mathrm{C}$ e D), de maneira dependente de concentração e equipotente. Estes efeitos foram reversíveis em cerca de 30 minutos após a retirada dos extratos da cuba (dados não mostrados).

Efeito dos extratos SM-MeOH e SM-AcOEt sobre as contrações tônicas induzidas por $40 \mathrm{mM}$ de $\mathrm{KCl}$ ou por $1 \mu \mathrm{M}$ de histamina ou de acetilcolina em íleo isolado de cobaia

Os extratos $(0,1-750 \mu \mathrm{g} / \mathrm{mL}) \mathrm{SM}-\mathrm{MeOH}$ e SM-AcOEt relaxaram o íleo pré-contraído $(\mathrm{n}=5)$ com $40 \mathrm{mM}$ de $\mathrm{KCl}\left(\log \mathrm{E}_{50}=1,9 \pm 0,09\right.$ e $1,9 \pm 0,1 \mu \mathrm{g} /$ $\mathrm{mL}$, respectivamente), $1 \mu \mathrm{M}$ de histamina $\left(\log \mathrm{CE}_{50}=\right.$ $1,9 \pm 0,07$ e $1,7 \pm 0,08 \mu \mathrm{g} / \mathrm{mL}$, respectivamente) ou de acetilcolina $\left(\log \mathrm{CE}_{50}=1,9 \pm 0,02\right.$ e $1,7 \pm 0,09 \mu \mathrm{g} / \mathrm{mL}$, respectivamente) de maneira dependente de concentração e equipotente (Figura $2 \mathrm{~A}$ e B). Estes efeitos foram reversíveis em cerca de 30 minutos após a retirada dos extratos da cuba (dados não mostrados).

\section{DISCUSSÃO}

No presente trabalho investigou-se os efeitos dos extratos SM-MeOH e SM-AcOEt em músculo liso visceral, e o achado mais importante deste estudo é a demonstração pela primeira vez que as partes aéreas de Solanum megalonyx Sendtn. exercem efeito espasmolítico não seletivo em íleo isolado de cobaia. Portanto, os resultados aqui apresentados trarão uma grande contribuição para a farmacologia do gênero Solanum e por sua vez para a família Solanaceae.

Os extratos SM-MeOH e SM-AcOEt inibiram as contrações fásicas induzidas tanto por acetilcolina quanto por histamina em íleo isolado de cobaia (Figura 1). O fato de não haver nenhuma diferença estatística entre os valores de $\mathrm{CI}_{50}$ é sugestivo de que os extratos podem estar agindo em um passo comum da via de sinalização 
que leva à contração muscular pelos agentes contráteis utilizados. Além disso, esses resultados sugerem que o(s) metabólito(s) secundário(s) responsável por esse efeito podem estar presentes nos dois extratos.

A contração no músculo liso em resposta a vários agentes, é frequentemente composta por duas fases: um componente fásico rápido seguido por um lento mais sustentado, o componente tônico (Bolton, 1979). Esta resposta bifásica é devido à fonte dupla de $\mathrm{Ca}^{+2}$ no músculo liso. Em íleo de cobaia os agonistas muscarínicos produzem esta resposta bifásica, e é sugerido que a contração fásica é causada por liberação de $\mathrm{Ca}^{+2}$ dos estoques intracelulares mediados por $\mathrm{IP}_{3}$ (Abdellatif, 1989; Kobayashi et al., 1989). Por outro lado, a contração tônica induzida por agonistas muscarínicos em íleo de cobaia é atribuída ao influxo de $\mathrm{Ca}^{+2}$ através dos canais de $\mathrm{Ca}^{+2}$ operados por voltagem $\left(\mathrm{Ca}_{\mathrm{v}}\right)$, uma vez que a contração tônica é suprimida pelo bloqueador de $\mathrm{Ca}_{\mathrm{v}}$, verapamil (Jim et al., 1981). A probabilidade de abertura dos $\mathrm{Ca}_{\mathrm{v}}$ é favorecida por uma despolarização de membrana por estímulo mediado por acoplamento fármaco e eletromecânico (Rembold, 1996).

Com o objetivo de verificar se os extratos $\mathrm{SM}-\mathrm{MeOH}$ e SM-AcOEt estariam agindo em nível de influxo de $\mathrm{Ca}^{+2}$ através da membrana plasmática em íleo de cobaia, avaliou-se seus efeitos sobre o componente tônico da resposta contrátil induzida por $\mathrm{KCl}$ (acoplamento eletromecânico) e por histamina ou acetilcolina (acoplamento fármaco e eletromecânico), onde foi observado que os extratos inibiram de maneira dependente de concentração e equipotente as contrações tônicas induzidas pelos três agentes contráteis (Figura 2). Esses resultados são sugestivos de que os extratos podem estar promovendo efeito relaxante em íleo isolado de cobaia por bloquear o influxo de $\mathrm{Ca}^{+2}$ via $\mathrm{Ca}_{\mathrm{v}}$, no entanto outros estudos são necessários para comprovar esta hipótese.

Pode-se concluir que os extratos SM-MeOH e SM-AcOEt, obtidos das partes aéreas de Solanum megalonyx Sendt, possuem metabólitos secundários com atividade espasmolítica não seletiva em íleo isolado de cobaia, em relação aos agentes contráteis testados.

\section{REFERÊNCIAS}

Abdellatif AA 1989. Calcium mobilizing receptors, polyphosphoinositides, generation of second messengers and contraction in mammalian smooth muscle: historical perspectives and corrent status. Life Sci 45: 757-786.

Abraham Z, Bhakuni SD, Garg HS, Goel AK, Mehrotra BN, Patnaik GK 1986. Screening of Indian plants for biological activity. Part XII. Indian J Exp Biol 24: 4868.

Almeida ER, Santos ER, Lins CFB, Mello AC, Souccar C, Lapa AJ 1984. Presença da acetilcolina no fruto de Solanum melongena L. Rev Inst Antibiotico 22: 113-120.

Barbosa-Filho JM, Agra MF, Oliveira RAG, Paulo MQ,
Troling G, Cunha EVL, Ataide JR, Bhattacharyya J 1991. Chemical and pharmacological investigation of Solanum species of Brazil - a search for solasodine and other potentially useful therapeutic agents. Mem I Oswaldo Cruz 86:189-191.

Barbosa-Filho JM, Vasconcelos THC, Alencar, AA, Batista LM, Oliveira RAG, Guedes DN, Falcão HS, Moura MD, Diniz MFFM, Modesto-Filho J 2005. Plants and their active constituents from South, Central, and North America with hypoglycemic activity. Rev Bras Farmacogn 15: 392-413.

Barbosa-Filho JM, Piuvezam MR, Moura MD, Silva MS, Lima KVB, Cunha EVL, Fechine IM, Takemura OS 2006. Anti-infl ammatory activity of alkaloids: A twentycentury review. Rev Bras Farmacogn 16: 109-139.

Bhakuni OS, Dhar ML, Dhar MM, Dhawan BN, Mehrotra BN 1969. Screening of Indian plants for biological activity. Part II. Indian J Biol 7: 250-262.

Bolton TB 1979. Mechanism of action of transmitters and other substances on smooth muscle. Physiol Rev 59: 606718 .

Boyd LJ 1928. Pharmacology of the homeopathic drugs. J Amer Ins Homeopathy 21: 209.

Braga FC, Valadares YM, Costa MA, Lombardi JA, Oliveira AB 2003. Estudo fitoquímico de Erythraea centaurium, Jacaranda caroba, Remijia ferruginea e Solanum paniculatum visando identificar marcadores químicos para o fitoterápico Ierobina ${ }^{\circledR}$. Rev Bras Farmacogn 13(Supl. 1): 28-31.

Cavalcante FA 2001. Mecanismo de ação espasmolitica de solavetivona, sesquiterpeno isolado das partes aéreas de Solanum jabrense Agra \& Nee (Solanaceae). João Pessoa, 81p. Dissertação de Mestrado - Programa de Pós-Graduação em Produtos Naturais e Sintéticos Bioativos, Universidade Federal da Paraíba.

Claudino FS, Medeiros AFD, Silva TMS, Carvalho MG, Braz-Filho R, Silva BA 2001. Avaliação do efeito espasmolítico dos extratos metanólico e metanólico aquoso de Solanum jabrense Agra \& Nee. XVI Reunião Anual da Federação de Sociedades de Biologia Experimental. Caxambú, Brasil.

Claudino FS 2003. Atividade espasmolitica de extratos obtidos de Solanum jabrense Agra \& Nee (Solanaceae). João Pessoa, 113p. Dissertação de Mestrado - Programa de Pós-Graduação em Produtos Naturais e Sintéticos Bioativos, Universidade Federal da Paraíba.

Friedman M, Rayburn JR, Bantle JA 1991. Developmental toxicology of potato alkaloids in the frog embryo teratogenesis assay - Xenopus (FETAX). Food Chem Toxicol 29: 537-547.

Jenkinson DH, Barnard EA, Hoyer D, Humphrey PPA, Leff P, Shankley NP 1995. International union of pharmacology committee on receptor nomenclature and drug classification. IX. Recommendations on terms and symbols in quantitative pharmacology. Pharmacol Rev 42: 255-266.

Jim K, Harris A, Rosenberger LB, Triggle DJ. 1981. Stereoselective and non-stereoselective effects of D 600 (methoxyverapamil) in smooth muscle. Eur $J$ Pharmacol 76: 67-72.

Kobayashi S, Kitazawa T, Somlyo AV, Somlyo AP 1989. Cyitosolic heparininhibits muscarinic and $\alpha$-adrenergic $\mathrm{Ca}^{+2}$-release in smooth muscle: physiological role of 
inositol 1,4,5-triphosphate in pharmacomechanical coupling. J Biol Chem 264: 17997-18004.

Nee, M 2001. Solanaceae Systematics the $21^{\text {sT }}$ Century. In:Van Der Berg RG, Barendse GWM; Van Der Weerdsen CM. (eds). Solanaceae V. Advances in Taxonomy and Utilization, Nijmegen University Press, p.3-22.

Rembold CM 1996. Biochemistry of Smooth Muscle Contraction In: Bárány, M. (org.) Electromechanical and pharmacomechanical coupling. San Diego: Academic Press, p.227-239.

Ribeiro EAN 2001. Estudo das ações cardiovasculares da fração aquosa do extrato etanólico do caule de Solanum stipulaceum Roem \& Schult. (Solanaceae) em ratos. João Pessoa, 101p. Dissertação de Mestrado - Programa de Pós-Graduação em Produtos Naturais e Sintéticos Bioativos, Universidade Federal da Paraíba.

Ribeiro EAN, Batitucci MCP, Lima JAT, Araújo IGA, Mauad H, Medeiros IA 2002. Cardiovascular effects induced by the aqueous fraction of the ethanol extract of the stem of Solanum stipulaceum in rats. Rev Bras Farmacogn 12 (Supl. 1): 34-35

Ribeiro R, Fiuza de Melo MMR, Barros F, Gomes C, Trolin G 1986. Acute antihypertensive effect in conscious rats produced by some medical plants used in the state of São Paulo. J Ethnopharmacol 15: 261-269.

Santos RF, Medeiros AFD, Alarcón KM, Sousa JP, Claudino FS, Cavalcante FA, Silva TMS, Silva BA 2003. Estudo comparativo do efeito espasmolítico entre Solanum agrarium Sendtner e Solanum stipulaceum Roem \& Schult. In: Souza, M.F.W. (org.) Iniciados. $9^{\text {a }}$ série. João Pessoa: Editora Universitária/UFPB, p.99-114.

Shum OL, Chiu KW 1991. Hypotensive action of Solanum melogena on normotensive rats. Phytother Res 5: 7681.

Silva JLV, Costa VCO, Cavalcante FA, Silva CC, Silva TMS, Agra MF, Silva BA 2000. Avaliação da atividade espasmolítica de Solanum paludosum Moric. (Solanaceae). XVI Simpósio de Plantas Medicinais do Brasil. Recife, Brasil.

Silva TMS, Silva CC, Braz-Filho R, Carvalho MG, Silva MS, Agra MF 2002. Constituintes químicos do extrato acetato de etila das partes aéreas de Solanum paludosum Moric. Rev Bras Farmacogn 12(Supl 1): 85-86.

Silva TMS, Agra MF, Jnanabrata Bhattacharyya J 2005. Studies on the alkaloids of Solanum of northeastern Brazil. Rev Bras farmacogn 15: 292-293.

Sun YD, Benishin CG 1994. $\mathrm{K}^{+}$channel openers relaxe longitudinal muscle of guinea-pig ileum. Eur $J$ Pharmacol 271: 453-459. 